\title{
Educación como fenómeno sociocultural
}

\author{
doi: 10.33264/rpa.202001-03 \\ Alexis Valenzuela Mayorga \\ Facultad de Ciencias de la Salud, Universidad Central de Chile \\ Diego Silva Jiménez \\ Facultad de Ciencias de la Salud, Universidad Central de Chile
}

\section{Resumen}

La relación entre educación y sociedad ha sido mediada constantemente por variables que se conjugan entre ellas mismas, como lo son la cultura, el contexto socio histórico, con miradas desde la psicología, sociología, ciencias de la educación y cultura, muchas veces entremezclándose, sin tener claridad cómo es que se configuran estas proyecciones. Se procederá a revisar desde una amplia mirada quien tiene la ventaja a la hora de plantear la norma dentro de la sociedad, develando la naturalización e invisibilización de la hegemonía. Revisando su interacción entre estas distintas aristas e intentando comprender la interacción cultura, sociedad y educación.

Palabras clave: educación, sociedad, cultura, historia.

\begin{abstract}
The relationship between education and society has been constantly mediated by variables that combine among themselves, such as culture, the socio-historical context, with views from psychology, sociology, education and culture, often intermingling, without being clear how is that these projections are configured. That is why we will proceed to review from a broad perspective who has the advantage when it comes to setting the standard within society. Reviewing their interaction between these different edges and trying to understand the interaction between culture, society and education.
\end{abstract}

Keywords: education, society, culture, history. 


\section{Introducción}

La educación como fenómeno cultural presenta una serie de visones tales como un aporte de culturalización para la humanidad (Álvarez, 2005), una institución de dominación similar a la Familia, la Iglesia y el Estado (Bourdieu, 2000), una forma de control social similar al panóptico (Foucault, 2012) o la oportunidad del pleno desarrollo del ser humano (Rogers 2011) y múltiples visiones desde la formalidad e informalidad, y su objetivo social.

Cuando hablamos de sociedad, hablamos de educación, cuando hablamos de valores, hablamos de educación, cuando hablamos de desarrollo hablamos de educación, cuando hablamos de socialización hacia nuevas generaciones, hablamos de educación, cuando hablamos de naturaleza del ser humano, hablamos de educación, cuando hablamos de cultura, hablamos de educación. (Navarro, 2019; Durkheim, 1998; Foucault, 2012; Silva, 2014; Caride, Gradaílle, \& Caballo, 2015).

La Teoría Dialéctica de la Construcción Social de la Realidad afirma que entre el individuo y la realidad social se da una relación dialéctica; el individuo (colectivo) crea la realidad social; pero, esta realidad social, objetivada, crea al individuo. La relación entre hombre (productor) y mundo social (producto) es dialéctica; ser humano (no aislado, sino en comunidades) y mundo social interactúan; el producto vuelve a actuar sobre el productor; la sociedad es un producto humano, es una realidad objetiva; así que el hombre es un producto social. (Yañez, 2010)

De esta manera se podría plantear que la educación es la que define la naturaleza del ser humano, sin embargo, podría ser todo lo contrario en donde la naturaleza del hombre define y perpetúa la educación a través de la cultura y contextos socio históricos. Es esta la disyuntiva que se intentara abordar en este artículo.

\section{Educación y Cultura}

Para Ricardo Nassif (1986) la sociedad es considerada como un hecho relacional que se consuma mediante la comunicación y la participación de sus miembros en actividades del bien común y a este hecho de comunicarse se le atribuye el carácter educativo de la sociedad. Es interesante la ingenuidad del autor al definir la sociedad en pro del bien común, definición de carácter aristotélica, muy lejano de posturas más críticas como las de Marx (2014) que entiende la sociedad como una estructura heterogénea de clases sociales en conflicto o Weber (2014) que la comprende desde un sistema de poder que permea todos los niveles de la sociedad, de las relaciones de los gobernados y los gobernantes, incluso Durkheim (citado por Lorenc, 2014) que reconoce en la sociedad la coerción social del individuo, forzado a seguir normas sociales que le son impuestas desde su nacimiento y que no tiene poder para 
modificar.

Esta relación sociedad-individuo es comprendida desde el Humanismo Rogeriano como personalización, esencia del proceso educativo, cuyo principal argumento posiciona al ser humano en el centro de la educación y la búsqueda del máximo desarrollo de las capacidades humanas, como un proceso de transformarse en persona (Rogers, 2011). Es importante destacar que Rogers da gran importancia a la relación y a la apertura que tenga el educador con el educando, a lo cual le llama proceso de empatía (Rogers, 1996). Así mismo Freire (2008), humanista latinoamericano trae un mensaje esperanzador de profunda fe en el hombre y su proceso de liberación (Freire, 2008), centrado en la relación dialéctica educador educando, donde ambos aprenden del otro, en base a una relación horizontal y colaborativa, que inicia con la liberación del individuo para continuar con la transformación de la sociedad, similar a los planteamientos de Maturana (citado por Mazorco, 2010) biólogo y construccionista que enfatiza el proceso de autoformación y adaptación autopoiética del individuo, siendo fundamental la relación afectiva entre estudiante y profesor, así como la emocionalidad del aprendizaje. Por lo tanto, los seres humanos no se educan solo, aunque tengan una capacidad innata de aprendizaje, sino que es fundamental el tipo de relación que se establezca entre estos 2 actores fundamentales: el educador y el educando, siempre en un contexto social e histórico determinado.

Por otra parte, es posible observar una concepción naturalizada y androcentrista de la educación (Bourdieu, 2000), invisibilizando a las mujeres como parte de la especie humana, e insertándola forzadamente en la categoría hombre. Una dificultad similar tuvo Freire (2008) cuando planteaba la liberación del hombre mediante la educación como práctica de libertad, produciendo un quiebre en sus discípulos y discípulas del movimiento de la educación popular, naciendo posteriormente el movimiento de educación popular feminista (Sáenz y Rapacc, 2018).

De esta manera es posible preguntase ¿Es la educación, un contexto socio-histórico, como perpetuación y conservación del acervo cultural por parte de la sociedad?

Esta interrogante es tremendamente controvertida, ya que devela una intencionalidad positiva en la sociedad, escondiendo la dominación y sometimiento de los seres humanos, en un proceso de uniforme continuidad de los valores funcionales y subordinación de lo distinto, lo raro, lo loco, lo enfermo, actuando la educación como un aparato represivo del ser humano, aplastando la expresión de lo diverso. (Foucault, 2012)

Pero la educación también tiene su dicotomía formalidad - informalidad, pudiendo 
generar procesos educativos de:

Educación formal o convencional: Que tienen por objetivo la transmisión de contenidos, valores, conocimiento desde la escuela e institucionalidad de las organizaciones formadoras (Álvarez, 2005).

La educación no formal: Un intermedio entre la educación formal y no formal, comprendiendo toda actividad educativa organizada sistemática impartida fuera del marco del sistema formal, para suministrar determinado aprendizaje a subgrupos concretos de la población (Colom, 2005) y la educación informal, que según Sanvisens es "considerada la educación de una persona como un universo de sucesos educativos acontecidos en diferentes tiempos.... con un grado de intencionalidad mínimo por parte del que educa y un grado de conciencia también mínimo por parte del ser que se educa" (Rubio E, 2008, p 16).

El uso de los conceptos no formal e informal por muchos años fue considerado sinónimos. Lo interesante es que la informalidad tendría una gradualidad desde una educación de mínima informalidad cuando el que educa tiene conciencia de su acción y el que recibe la educación no, hasta una educación máxima de informalidad, cuando el que educa no tiene intencionalidad, ni el que es educado tiene conciencia. (Berberl y Díaz, 2014).

\section{Educación y naturaleza del ser humano: ¿Quién define a quién?}

"La educación es un proceso humano y cultural complejo" (León 2007, p595) del mismo modo una teoría educativa no tiene un origen arbitrario, sino es consecuencia y producto de una determinada concepción del mundo y de la vida (Hernández, 2018)

Cuando hablamos de la naturaleza del ser humano, hablamos del desarrollo de la sociedad, es aquí donde resulta difícil no hablar de ciertos factores que inciden o predisponen de esta. La sociedad se enmarca en los contextos históricos, psicológicos y sociológicos, todo ello influye y produce tanto un sentido y otro conduce y desarrollan la educación (Figura $\mathrm{N}^{\circ}{ }^{1}$ )

La incidencia de la educación en la sociedad logra traspasar los muros de las instituciones educativas y nos invita a realizar un profundo análisis sobre su entorno tanto próximo como lejano (Meza y Páez, 2016)

Por otra parte, Ruiz, 2013 plantea que la educación es reconstrucción de la experiencia, proceso de adaptación del ambiente, socialización de las nuevas generaciones y desenvolvimiento de la personalidad a través de la cultura.

Así mismo Martínez (2008) y Stefoni, Stang y Riedemann (2016) cuestionan en sus escritos que exista un concepto verdadero de educación si no se asienta sobre una 
imagen de la persona humana, convicción que encierra sus raíces en la vida cultural.

Figura №1 Influencia de la Sociedad en la Educación.

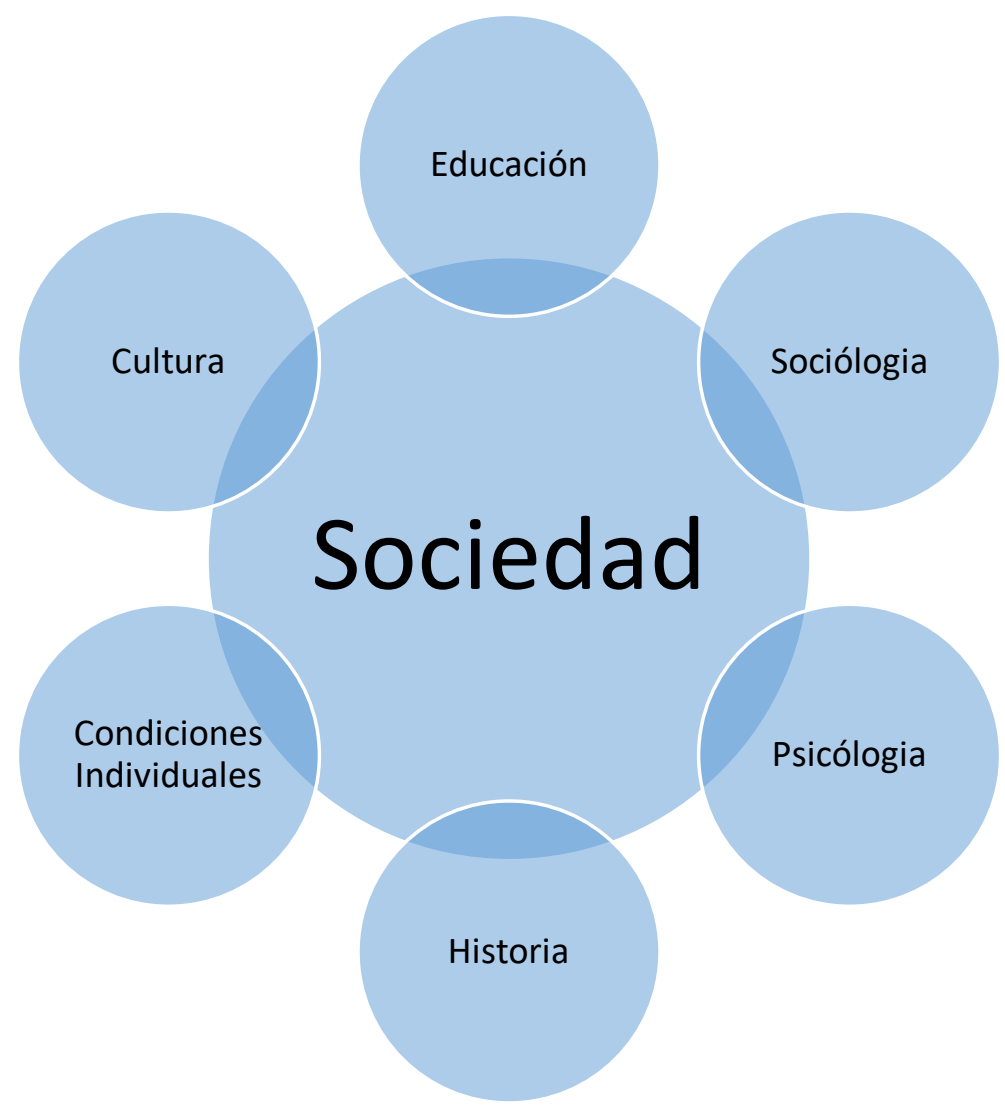

Fuente: elaboración propia.

La educación se caracteriza, como un fenómeno social complejo, Durkheim (1998) se refiere a ello de la siguiente manera "la educación común es función del estado social; pues cada sociedad busca realizar en sus miembros, por vía de la educación, un ideal que le es propio.

Cabrera y Vásquez (2012, p3) “Plantean que el ser social determina la conciencia social” y se puede agregar que esto sucede a través de la educación. Sin embargo, este desarrollo y relación va mucho más allá de lo planteado, pues, es inconcebible una pedagogía como teoría de la educación sin una filosofía del ser humano, que se plantee, ¿Qué es el ser humano? ¿Cómo debe ser? Y ¿Cuál es su destino? (Ortiz ,2017).

De la misma forma lo plantea (Bruner 1997 y Vigotsky 1978 citados en León, 2007, p600) tanto "hombre y mente son hechuras de la cultura. La mente es producto de 
la educación. También los valores, los afectos, las emociones, el carácter, el conocimiento, la cognición, las particularidades del cuerpo son obras de la cultura, de la educación"

Sin embargo, a pesar de estas afirmaciones, aun no podemos responder ¿Como la educación define la naturaleza del ser humano? En este sentido aparece una el fenómeno de la triada y desarrollo de vida humana. El humano en base a la educación y la sociedad crean y desarrollan su cultura. (Figura $\mathrm{N}^{\circ} 2$ )

Figura N² Relación Sociedad- Educación.

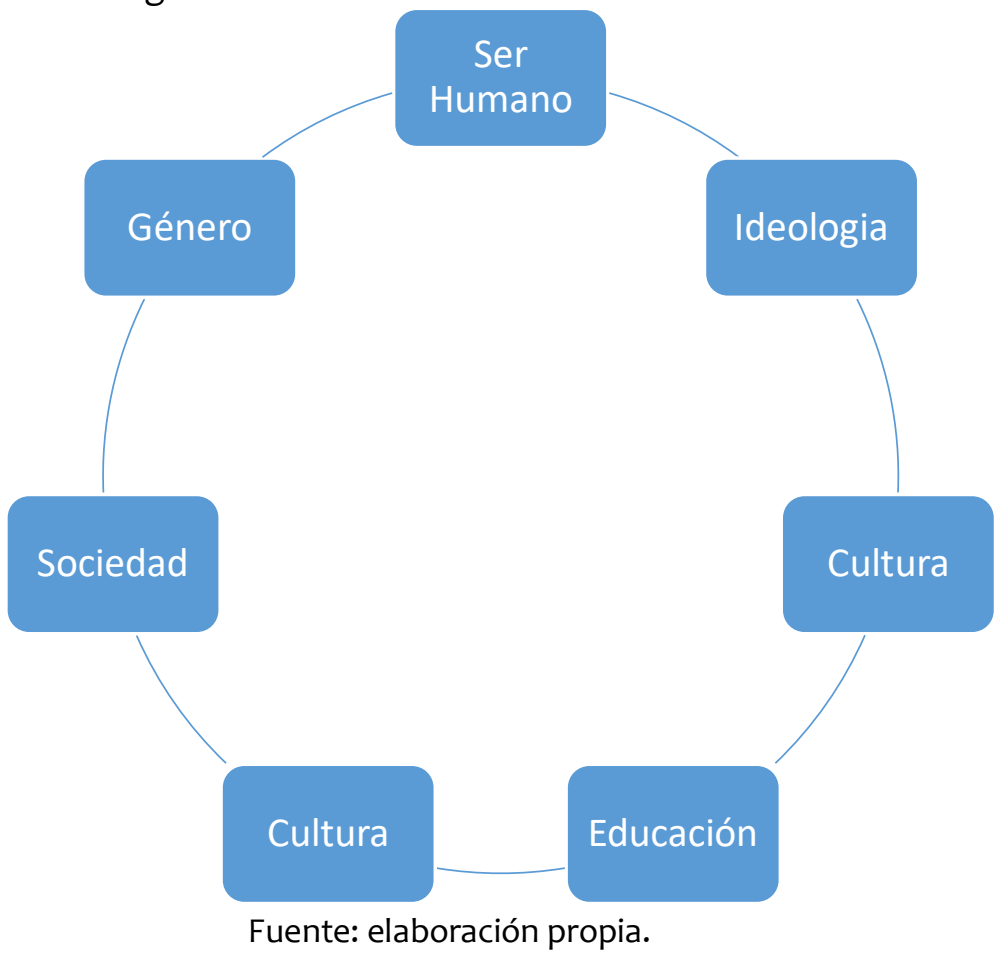

Frente a esto, la clave para diversos autores más que el sistema formal de educación es el sistema no formal. Es en este sistema donde se enseñan, valores tales como; honor, lealtad, medio ambiente, civismo, entre otros. (Berberl y Díaz, 2014 \& Colom, 2005)

Entonces, si la educación se debe complementar con educación no formal, esta educación no formal se puede conseguir en la sociedad, es decir desde la cultura hacia la educación y desde la educación hacia la sociedad.

Es por esto que la "educación no se puede deshacer de la cultura y de la tradición. Una de sus tareas es mantener incólumes la cultura y la tradición y expresar los valores que la cohesionan para pensar y para la acción de los sistemas sociales" (León, 2007, p600) 
Sin embargo, la educación en Platón, Plutarco o Pestalozzi ya era vocacionalmente social: hay que educar a la ciudad o al pueblo para que llegue a darse una verdadera educación del individuo (Caride, Gradaílle, \& Caballo, 2015)

De esta manera podemos entender ciertos desarrollos sociales en base a la educación del ser humano, esta desde su mismo ethos, creencias y verdades, ejemplo de ello es Oriente, gran imperio que conocía la pólvora mucho antes que nadie y no la utilizo con fines bélicos, sino con fines recreativos, sin embargo, la guerra y conflicto de sus países vecinos de Occidente fue lo que los llevo a su desarrollo (Silva, 2014), claro resultado ambos de su educación, cultura y sociedad.

Por ende, es complejo poder decir quién y cómo influye en que, si la educación en la naturaleza del ser humano o al revés, ni como los flujos continuos y consientes de influencias afectan el uno al otro, en algunos tiempos es la naturaleza del ser humano quien rige la cultura (educación) y otras veces es la cultura (educación) quien quiere determinar la naturaleza de la persona humana.

Pero creer que la cultura es un ente que meramente podemos describirlo resulta inocente, ya que esta integra los intereses políticos, económico valóricos e ideológicos de una elite, que impondrá sus cosmovisiones a la sociedad para naturalizar la dominación, desde la formación inicial hasta la selección de la disciplina profesional, asignando atributos de género a las distintas ocupaciones, dejando la educación, el cuidado, la rehabilitación e intervención social a la esfera de lo femenino con profesiones como Enfermería, Terapia Ocupacional, Trabajo Social, Educación de Párvulos, Pedagogía y otras; así como la productividad, las llamadas ciencias duras, las matemáticas y el derecho a lo masculino con disciplinas como Ingeniería, Química, Medicina, Física, Derecho y otras (López Senz M, 1995). Incluso aquellos que rompen con los estereotipos de género y estudian una profesión distinta a la socialmente asignada a su sexo, se encontraran con distintos procesos de discriminación, en el caso de los varones una discriminación positiva de parte de sus pares femeninas y maestros, empujándolos a la promoción y ocupación de posiciones de poder en el desarrollo de su disciplina (Valenzuela y De Keijzer, 2016), en cambio las mujeres encontraran una discriminación negativa, de acoso de sus pares masculinos y barreras tales como el techo de cristal (Alizade y Seeling, 2007): un conjunto de obstáculos invisibles que le impedirán seguir ascendiendo en el ámbito laboral. 


\section{Conclusión}

El hecho educativo es comprendido desde una diversidad de posiciones dando mayor o menor énfasis al contexto social y su poder de moldeamiento de los seres humanos mediante la educación. Esta puede ser formal informal o no formal, con diversidad de grados de intencionalidad y conciencia entre educador y educando.

Entonces para responder la pregunta ¿Como la educación define la naturaleza del ser humano? Debemos concluir lo siguiente.

Es la educación quien desarrolló a las sociedades (Silva, 2014), la sociedad a través de la educación ha generado y perpetuado prácticas, en contra de las mujeres, llevándolas a un lugar secundario en la sociedad, en el ámbito laboral y sexual y hoy en plena confrontación de dicha situación, la educación ha hecho esfuerzos en pro de la igualdad, que no han logrado adecuarse a dicha contingencia, multiplicando los privilegios masculinos y los formatos androcentristas de las instituciones educacionales (Valenzuela, De Keijzer, 2016). Por lo que nos genera más dudas que certezas en esta conclusión.

¿Cómo confirmar o negar esto? Es complejo en esta reflexión, pero podemos plantear, que este sistema bidireccional no tiene un ente a tiempo completo, a veces la naturaleza del ser humano es quien se revela o más bien rebela y otras, la educación (cultura) y cuando uno influye el otro/ otra se subyuga.

En otras palabras "La educación no puede soslayar ni ignorar la cultura” (León, 2007, p600) ya que es parte de su producción y reproducción.

\section{Referencias}

Alizade y Seeling (2007). El techo de cristal: Perspectivas psicoanalíticas sobre mujeres y el poder. Lumen.

Alvarez, R. (2005). Educación para la Salud. Manual Moderno.

Bourdieu, P. (2000). La dominación masculina. Anagrama.

Cabrera, I. y Vásquez J. (2012) La educación un fenómeno social complejo. Revista digital de sociedad de la información $N^{\circ} 38$, 1-6. http://www.sociedadelainformacion.com/38/complejo.pdf

Caride, J., Gradaílle, R., \& Caballo, M. (2015). De la pedagogía social como educación, a la educación social como Pedagogía. Perfiles educativos, 37(148), 04-11. http://www.scielo.org.mx/scielo.php?script=sci_arttext\&pid=S0185-

26982015000200016\&lng=es\&tlng=es

Revista Pensamiento Académico de la Universidad UNIACC

Vol. $3 \mathrm{~N}^{\circ} 1,2020$ 
Colom Cañellas, A.J. (2005). Continuidad y Complementariedad entre La Educación Formal Y No Formal, Revista de Educación, núm. 338, 9-22 http://www.revistaeducacion.educacion.es/re338/re338_03.pdf

Berbel Gómez ,N y Díaz Gómez, M, Educación formal y no formal. Un punto de encuentro en educación musical, Aula Abierta 42 (2014) 47-52. https://ac.elscdn.com/S0210277314700083/1-s2.0-S0210277314700083-main.pdf?.tid=3fd363ee-7b284598-9c96-9cd8923ccf4f\&acdnat $=1536448475 \_80 d 92$ bcd70319f3b65ea38648e 363 e6o

Durkheim, Émile. (1998) Educación y pedagogía. Ensayos y controversias. Editorial Losada.

Foucault, M. (2012). Vigilar y castigar. Biblioteca Ediciones Nueva Siglo XXI

Freire, P (2008). Educación como práctica de la libertad. Editorial Siglo XXI.

Hernández, A. «Teoria de l'evolució i modelització pedagògica: una perspectiva ecològica». Temps d’Educació, [en línia], 2018, Núm. 55, p. 171-90, https://www.raco.cat/index.php/TempsEducacio/article/view/348748

León, Aníbal (2007). Qué es la educación. Educere, 11(39), 595-604 http://www.redalyc.org/articulo.oa?id=35603903

López Senz, Mercedes (1995). La elección de una carrera típicamente femenina masculina, desde la perspectiva psicosocial: La influencia del género. Ministerio de Educación y Ciencia.

Lorenc Valcarce, Federico. (2014). Émile Durkheim y la teoría sociológica de la acción. Andamios, 11(26), 299-322.

http://www.scielo.org.mx/scielo.php?script=sci_arttext\&pid=S1870-

$00632014000300012 \& \operatorname{lng}=$ es\&tlng=es.

Martínez García, Bernardo. (2008). El aprendizaje de la cultura y la cultura de aprender. Convergencia, 15(48),287-307.

http://www.scielo.org.mx/scielo.php?script=sci_arttext\&pid=S1405-

$14352008000300011 \&$ lng=es\&tlng=es.

Marx, C (2014) El capital: Crítica de la economía política, tomo I. De la ed. Ricardo Campa- $4^{\mathrm{a}}$ ed.

Mazorco (2010) Educación en crisis. Revista Polis, Caracas, Venezuela. Revista de la Universidad Bolivariana, 9, $\mathrm{N}^{\circ} 25$.

Meza,J. y Páez, R. (2016)Familia, escuela y desarrollo humano. Rutas de investigación educativa / Universidad de La Salle y CLACSO.

Nassif, R (1986). Teoría de la Educación. Editorial Cincel

Navarro, I. Q. (2019). Posthumanismo y educación. Ideas Educativas en Perspectiva 
Filosófica e Histórica.

Ortiz, A. (2017) Configuración epistémica de la pedagogía. Tendencias que han proliferado en la historia de la educación. XXRev. hist.edu.latinoam - Vol. 19 No. 29, julio - diciembre 2017 - ISSN: 0122-7238 - pp. 165-195

Rogers, C (1996). Libertad y creatividad en la Educación. Paidós.

Rogers, C (2011). El proceso de convertirse en persona. Paidós.

Rubio, E (2008). Aprendizaje a lo largo de la vida. Revista Participación educativa número 9. Consejo Escolar del Estado, Ediciones Ministerio de Educación Gobierno de España.

Ruiz, Guillermo (2013) La teoría de la experiencia de John Dewey: significación histórica y vigencia en el debate teórico contemporáneo. Foro de Educación, vol. 11, $\begin{array}{lllll}\text { núm. } & 15, \quad \text { enero-diciembre, } & \text { 2013, } & \text { 103-124 }\end{array}$ https://www.redalyc.org/pdf/4475/447544540006.pdf

Sáenz y Rapacc (2018). La educación popular feminista. Biblioteca digital. http://bdigital.unal.edu.co/45596/1/laeducaci\%C3\%B3npopularfeministauna.pdf.

Silva, D. (2014) Causas de la Divergencia entre occidente y el resto del mundo en la historia economía global. Diferencias entre los enfoques Cohen, Ferguson y $\begin{array}{llll}\text { Engerman-Sokoloff. } \quad \text { Revista } \quad \text { Akademeia } & N^{\circ} 2,2, & 111-126 .\end{array}$ http://repositorio.ugm.cl/bitstream/handle/12345/1386/Causas\%20de\%20la\%2odivergencia\%20 entre $\% 200$ ccidente $\% 20 \% \% 20$ el $\% 20$ resto $\% 20$ del $\% 20$ mundo\%20en $\% 20$ la $\% 20 \% 20$ historia\%20econ\% C3\%B3mica\%2oglobal.pdf?sequence=1\&isAllowed=y

Stefoni, Carolina, Stang, Fernanda, \& Riedemann, Andrea. (2016). Educación e interculturalidad en Chile: Un marco para el análisis. Estudios internacionales (Santiago), 48(185), 153-182. https://dx.doi.org/10.5354/0719-3769.2016.44534

Valenzuela y De Keijzer (2016). Hombres que estudian profesiones femeninas. Ediciones Universidad Central.

Vidal, Josep. (2013). La búsqueda de la realidad o de la verdad: una aproximación a partir de la teoría sociológica. Cinta de moebio, (47), 95114. https://dx.doi.org/10.4067/S0717-554X2013000200004

Weber, M (2014) Economía y Sociedad. Fondo de la Cultura.

Yañez, R. (2010) La construcción social de la realidad, la posición de Peter L. Berger y Thomas Luckmann. Ars Boni et Aequi. Vol. 6, No. 2, 2010, págs. 289-304 https://dialnet.unirioja.es/servlet/articulo?codigo $=3262960$ 


\section{Alexis Valenzuela Mayorga}

Trabajador Social, Universidad Metropolitana del Estado, Magister en Salud Pública, Universidad de Chile, Doctorando en Educación UPT, Perú, Diplomado en Docencia Universitaria, Universidad Central De Chile. Director del Centro de Salud Pública del Instituto de Investigación e Innovación en Salud de la Facultad de Ciencias de la Salud Universidad Central de Chile.

\section{Diego Silva Jiménez}

Administrador Público y Magister en Gerencia y Políticas Públicas, Universidad de Santiago de Chile, @Magíster en Ciencias de la Educación Mención Docencia e Investigación Universitaria, Universidad Central De Chile, Diplomado Para las Competencias Investigativas en Educación, Universidad SEK, Diplomado En Investigación Universitaria, Universidad Central De Chile. Coordinador Área de Gestión del Centro de Salud Pública del Instituto de Investigación e Innovación en Salud de la Facultad de Ciencias de la Salud Universidad Central de Chile. 